\title{
Effects of air humidity on the performance of a polymer insulator under lightning induced voltage conditions
}

\begin{abstract}
Lightning induced voltage is one of the important issues pertinent to line interruptions in distribution lines. The behaviour of a polymer insulator under different weather conditions may affect the overall stability of a power flow in the line. In this paper, the lightning induced voltage due to a typical sample of return stroke current on a distribution line has been investigated, and the electrical behaviour of a polymer insulator $(10 \mathrm{kV})$ under different air humidity conditions was analysed in detail. The results show that the air humidity has a direct effect on the electrical performance of a polymer insulator under lightning induced voltage conditions, which reduces the reliability of the system. Therefore, in designing new distribution lines and in modifying existing lines, various safety and protection parameters such as determining the critical distances around the distribution lines where lightning induced over voltage (LIOV) may occur, the humidity of air should be treated as an essential input parameter. Thus, the field propagation along an insulator based on recorded local information (weather conditions) can be helpful and should be taken into account in taking protection measures.
\end{abstract}

Keyword: Lightning electromagnetic fields; Lightning induced voltage; Polymer insulator 